01

\title{
Исследование переходного состояния и динамики фотохимических трансформаций молекул хромонов
}

\author{
() С.В. Гагарский ${ }^{1}$, Е.П. Гребенников ${ }^{2}$, В.В. Кийко ${ }^{1}$, К.С. Левченко ${ }^{2}$, А.Н. Сергеев ${ }^{1}$, \\ Я.Ю. Фомичева ${ }^{1, \uparrow}$, К. Oberhofer ${ }^{3}$, H. Iglev $^{3}$ \\ ${ }^{1}$ Университет ИТМО, \\ 197101 Санкт-Петербург, Россия \\ ${ }^{2}$ ЦНИТИ „Техномаш“, \\ 121108 Москва, Россия \\ ${ }^{3}$ Technical University of Munich, \\ 80333 Munich, Germany \\ ฯ e-mail: afuerra@gmail.com:
}

Поступила в Редакцию 16.05.2018 г.

\begin{abstract}
Представлены результаты исследования динамики фотопреобразования молекул хромонов методом спектроскопии переходного состояния. Измерены спектры изменения оптической плотности раствора соединений хромонов в толуоле в процессе фототрансформации в диапазоне сотен fs-сотен ps. Релаксация наведенного спектра поглощения описывается двухэкспоненциальной функцией с характерными временными постоянными порядка единиц пикосекунд и единиц наносекунд. Предложена схема динамики фототрансформации молекул хромонов, соответствующая наблюдаемой динамике спектров поглощения.
\end{abstract}

DOI: $10.21883 /$ OS.2018.10.46695.131-18

\section{Введение}

Светочувствительные органические соединения класса хромонов представляют интерес как с точки зрения изучения их фундаментальных свойств, так и для прикладных задач разработки систем оптической записи и хранения информации, в том числе для разработки многослойных флуоресцентных дисков [1]. Такие соединения при воздействии УФ излучения с длиной волны в области $250-350 \mathrm{~nm}$ необратимо преобразуются из исходного нелюминесцирующего состояния $A$ в стабильную форму $C$ через короткоживущее состояние $B$ (рис. $1, a)[2,3]$. У полученной в результате преобразования формы $C$ появляется полоса поглощения в спектральной области $400-500 \mathrm{~nm}$. При воздействии излучением в этой области спектра, преобразованные молекулы хромонов люминесцируют. В результате селекции по комплексу показателей, включая долговременную химическую и фотостабильность, а также квантовым выходам фотохимической реакции, из различных синтезированных соединений этого класса было выделено соединение 2-(фуран-2-ил)-3-(тиофен-2-карбонил)хромен-4-он (LHC-480) [2].

Соответствующие спектры поглощения в исходном и трансформированном состоянии, а также спектр люминесценции для формы $C$ представлены на рис. $1, b$.

Для практических применений [1] особый интерес представляет использование нелинейного режима записи. В работах [4,5] были приведены результаты измерения порогов нелинейной записи люминесцентных меток в соединениях хромонов, а также исследованы некоторые люминесцентные свойства этих соединений. Более детальное исследование механизмов и динамики фототрансформации молекул позволит выбрать оптимальные параметры записывающего источника лазерного излучения, а также сформулировать требования для модификации самих светочувствительных соединений и их свойств.

Одним из методов измерения быстрой (с разрешением до сотен фемтосекунд $[6,7])$ динамики фототрансформации молекул является спектроскопия переходного состояния, в частности спектроскопия поглощения, в которой измеряется быстрая динамика спектров поглощения образца („pump-probe“ спектроскопия). Такой метод широко используется для исследования динамики преобразования светочувствительных молекул [6-15].

\section{Методика эксперимента}

Схема экспериментальной установки приведена на рис. 2. Источником фемтосекундных импульсов являлся титан-сапфировый лазер с регенеративным усилителем „CPA-2010 Clark MXR“. Длительность импульса coставляла $150 \mathrm{fs}$, энергия - $900 \mu \mathrm{J}$, центральная длина волны $-799 \mathrm{~nm}$. Частота повторения импульсов составляла $1 \mathrm{kHz}$.

Излучение титан-сапфирового лазера разделялось на два канала. В одном канале формировался импульс возбуждения, инициирующий процессы фотопреобразования в молекуле, во втором - зондирующий импульс.

Для формирования импульса возбуждения излучение титан-сапфирового лазера проходило через оптический параметрический преобразователь (NOPA I, HORIBA SCIENTIFICN), обеспечивающий перестройку длины 


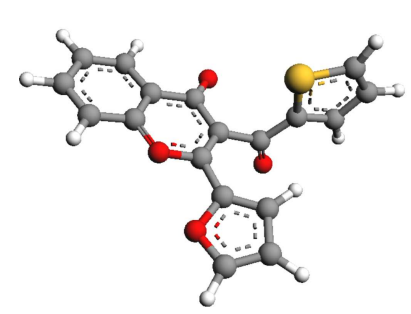

$A$

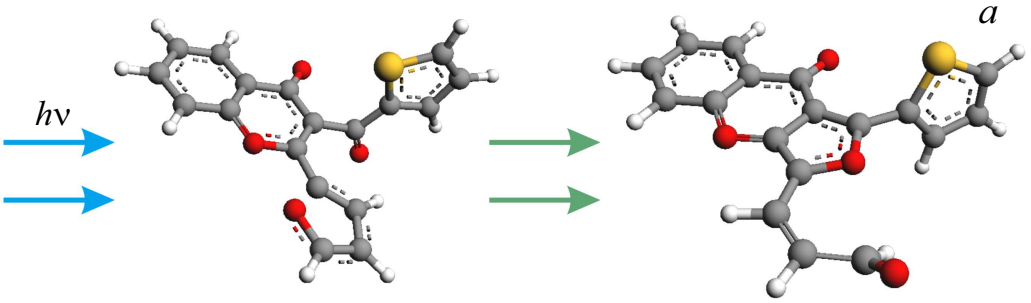

$B$

$C$ $b$

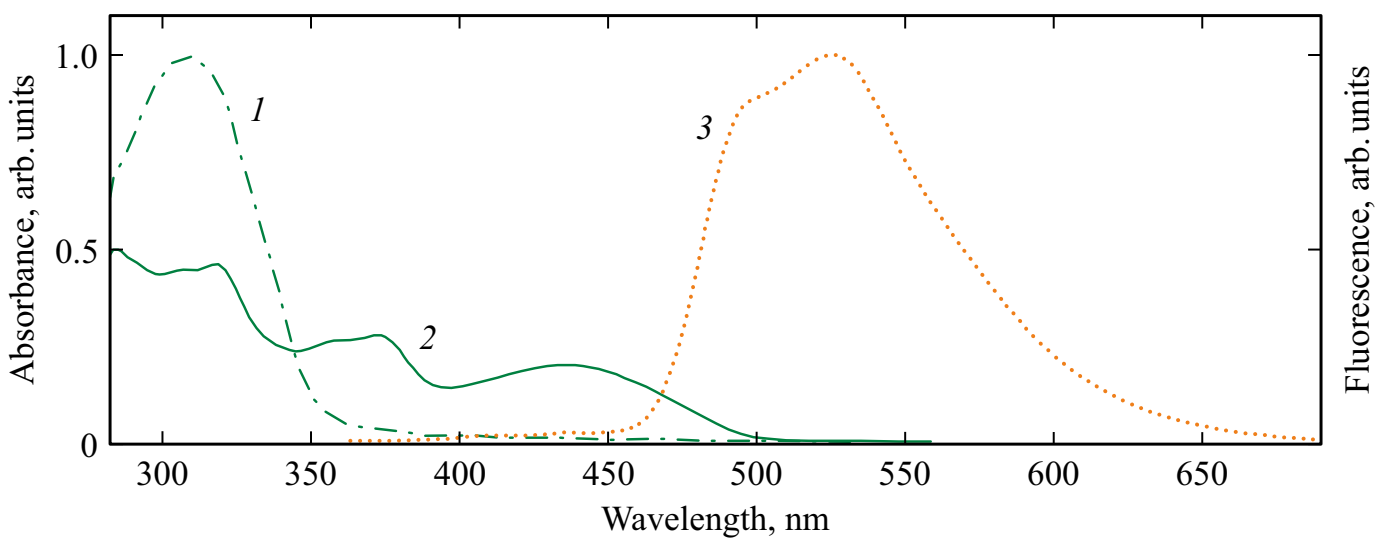

Рис. 1. Трансформация соединения LHC-480 при воздействии УФ излучения $(a)$. Спектры соединения LHC-480: 1 - спектр поглощения для состояния $A ; 2-$ спектр поглощения для состояния $C ; 3-$ спектр люминесценции для состояния $C(b)$.

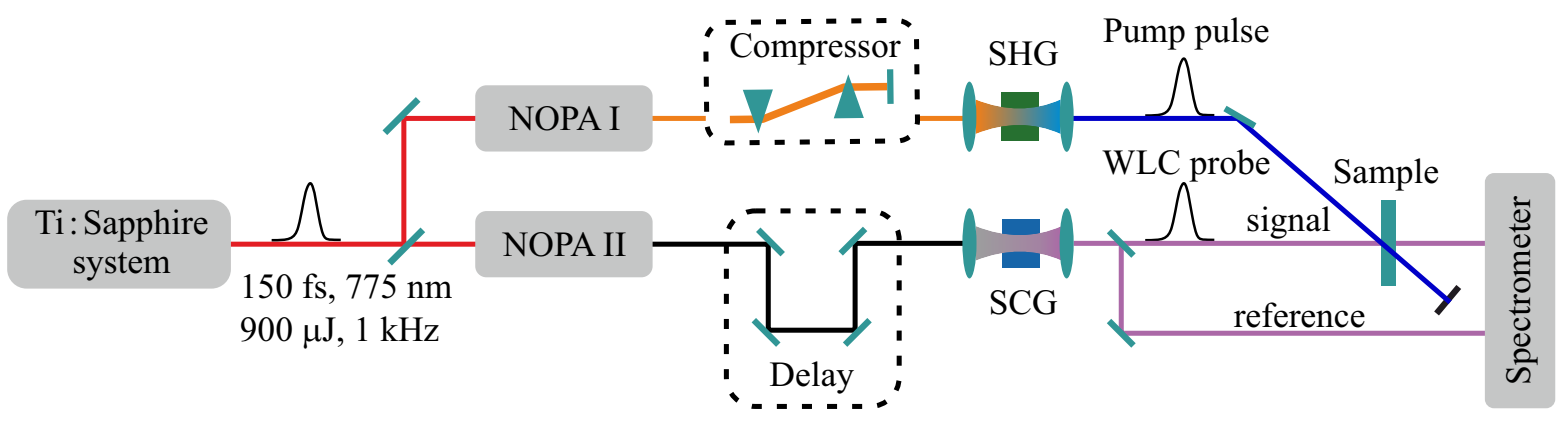

Рис. 2. Схема экспериментальной установки.

волны в диапазоне 550-1500 nm. Компрессор обеспечивал сокращение длительности фемтосекундного импульса до 30-50 fs. Для получения возбуждающего импульса в УФ диапазоне спектра использовался кристалл второй гармоники (BВО) толщиной $100 \mu \mathrm{m}$. Длительность полученного возбуждающего импульса составляла $50 \mathrm{fs}$, энергия в импульсе - $200 \mathrm{~nJ}$, длина волны $-320 \mathrm{~nm}$.

Во втором канале с помощью параметрического преобразователя (NOPA II, TOPAS LIGHT CONVERSION) формировался импульс с длиной волны $1500 \mathrm{~nm}$ и длительностью около $50 \mathrm{fs}$. Полученное излучение фокусировалось в сапфировую пластину толщиной $3 \mathrm{~mm}$ для формирования излучения суперконтинуума (WLC), перекрывающего диапазон длин волн от 450 до $1000 \mathrm{~nm}$. Полученное излучение суперкон- тинуума использовалось в качестве зондирующего импульса. Линия задержки (Delay) обеспечивала смещение зондирующего импульса относительно возбуждающего в диапазоне от сотен фемтосекунд до 500 ps. С помощью эталонов обеспечивалась перестройка задержки в диапазоне до $10 \mathrm{ps}$, в большем диапазоне (до 500 ps) - с помощью механической линии задержки.

Зондирующий импульс разделялся на опорный и сигнальный каналы. Пропускание образца регистрировалось с помощью спектрометра (Horiba Scientific).

В качестве образца использовался раствор молекул хромонов в толуоле с концентрацией 5 wt.\%. Раствор помещался в прокачиваемую кварцевую кювету толщиной $1 \mathrm{~mm}$. 


\section{Результаты эксперимента и их обсуждение}

Измерение спектров переходного состояния проводилось в два этапа. На первом этапе измерялась динамика спектров поглощения в диапазоне изменения задержек до $10 \mathrm{ps}$.

На рис. 3 представлены спектры изменения оптической плотности $\triangle O D$ образца для различных значений задержки между возбуждающим и зондирующим импульсами. Значение задержки - 1 ps соответствует начальному значению $\triangle O D$, т.е. значению до момента воздействия возбуждающего импульса. Для полученных зависимостей достоверными являются значения в области длин волн $450-1000 \mathrm{~nm}$, где соответствующие значения $\triangle O D$ близки к нулю.

На полученных спектрах видны характерные пики поглощения в области $450-500 \mathrm{~nm}$ и широкой полосы в области $600-1000 \mathrm{~nm}$.

В течение первой пикосекунды происходит быстрая релаксация пика поглощения области $450-500 \mathrm{~nm}$. В диапазоне задержек от 1 до 3 ps наблюдается смещение положения пика поглощения в более длинноволновую область.

На втором этапе эксперимента измерялась динамика спектров поглощения в большем диапазоне изменения задержек (до 500 ps), но с меньшим временным разрешением. Соответствующие зависимости приведены на рис. 4.

Как видно, на приведенных зависимостях наблюдается медленное продолжение релаксации спектров $\triangle O D$. Характерных изменений в форме спектра не наблюдается.

На рис. 5 показаны экспериментально полученные зависимости изменения оптической плотности от времени задержки для различных длин волн и их аппроксимация двухэкспоненциальной функцией (1):

$$
\Delta O D(t)=A_{1} \exp \left(-\frac{t}{\tau_{1}}\right)+A_{2} \exp \left(-\frac{t}{\tau_{2}}\right)
$$

где $\tau_{1}, \tau_{2}$ - временные постоянные релаксации.

В таблице приведены постоянные времени для различных длин волн. Характерные значения постоянных релаксации для большинства длин волн близки и составляют $\sim 1.5 \mathrm{ps}$ для быстрой компоненты и $\sim 1.5 \mathrm{~ns}$ для медленной. Существенное отличие в значениях постоянных релаксации наблюдается лишь для длины волны $500 \mathrm{~nm}$, т.е. в области, где наблюдается смещение положения пика поглощения на спектрах $\triangle O D$.

Для описания наблюдаемой динамики спектров может быть предложена схема возбуждения и трансформации молекул хромонов, энергетическая диаграмма для которой приведена на рис. 6. Поскольку в течение первой пикосекунды после возбуждения молекулы в спектрах наблюдается только релаксация наведенного при возбуждении поглощения, а в диапазоне от $1-1.5$ до 3-5 ps происходит смещение пика поглощения в

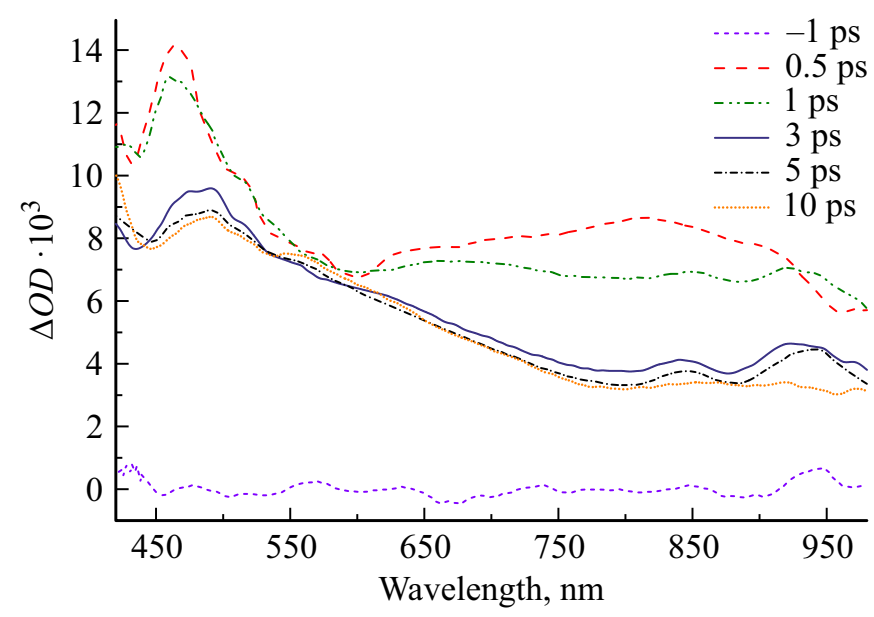

Рис. 3. Зависимость изменения оптической плотности $(\triangle O D)$ от длины волны для задержек до 10 ps.

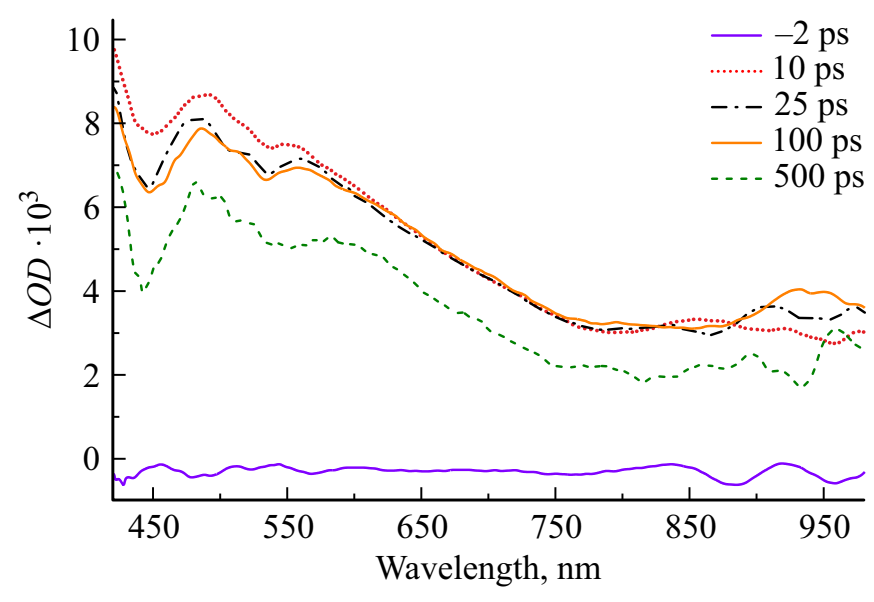

Рис. 4. Зависимость изменения оптической плотности $(\Delta O D)$ от длины волны для задержек до 500 ps.

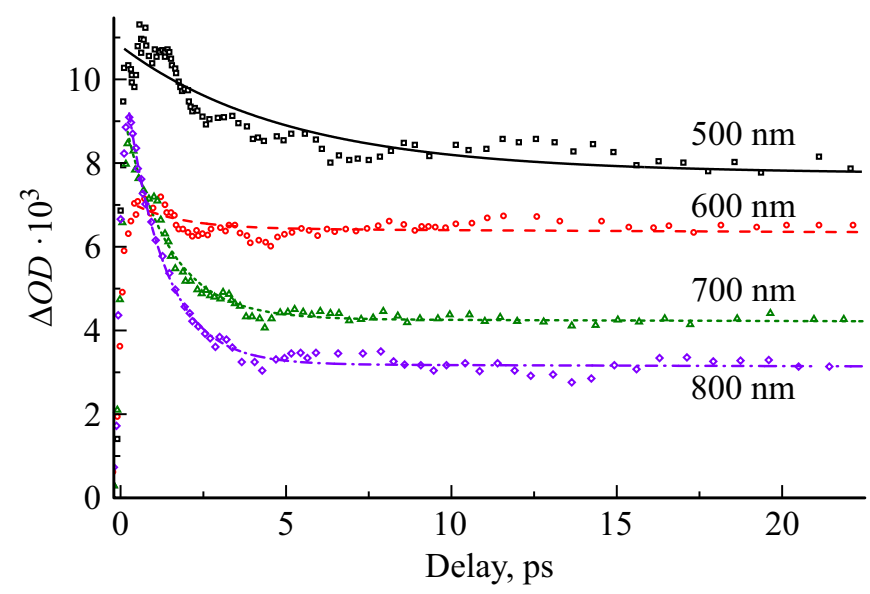

Рис. 5. Зависимость изменения оптической плотности $(\triangle O D)$ от задержки для различных длин волн. 


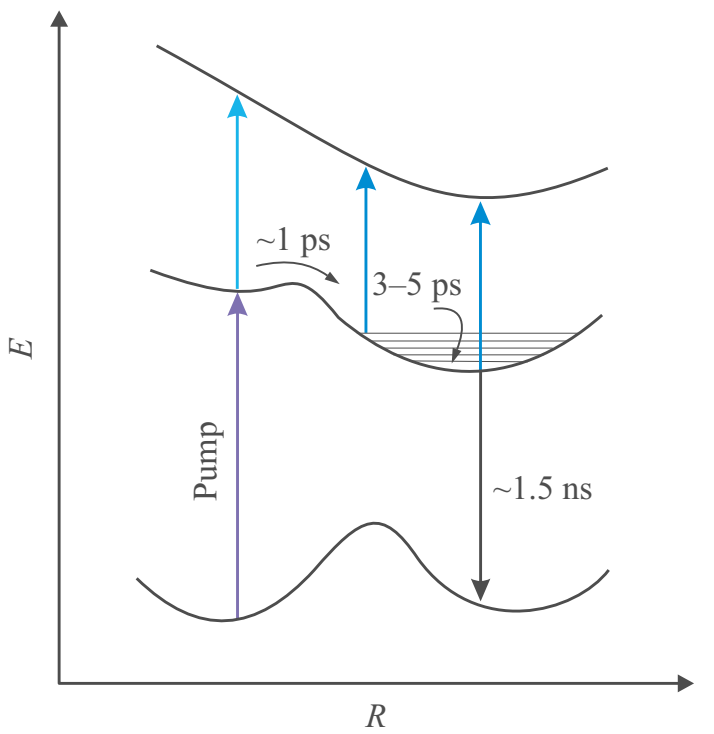

Рис. 6. Предполагаемая энергетическая диаграмма динамики трансформации молекул хромонов LHC480.

Постоянные времени релаксации наведенных спектров поглощения

\begin{tabular}{l|l|l}
\hline Длина волны, $\mathrm{nm}$ & $\tau_{1} \mathrm{ps}$ & $\tau_{2} \mathrm{ps}$ \\
\hline 500 & 4.99 & 2670 \\
600 & 1.44 & 1647 \\
650 & 1.32 & 1673 \\
700 & 1.32 & 1698 \\
750 & 1.31 & 1784 \\
800 & 1.14 & 1375
\end{tabular}

спектре, можно предположить существование некоторого потенциального барьера в возбужденном состоянии молекулы, на преодоление которого нужно время. Поэтому в течение $1-1.5 \mathrm{ps}$ мы видим релаксацию без каких-либо смещений.

После преодоления потенциального барьера наблюдается смещение пика поглощения в длинноволновую область, что можно связать с релаксацией электрона к новому минимуму потенциальной энергии. Данному возбужденному состоянию молекулы можно поставить в соответствие промежуточное состояние молекулы $B$ (рис. 1).

В дальнейшем с постоянной времени $\sim 1.5 \mathrm{~ns}$ наблюдается релаксация спектров поглощения возбужденного состояния, соответствующая переходу молекулы в конечное флуоресцирующее состояние $C$.

Описанной динамике, предположительно, соответствуют следующие процессы в молекуле: после возбуждения внешних электронов фотонами светового импульса накачки начинается процесс трансформации возникает перераспределение электронной плотности и происходит разрыв химической связи в одном из колец исходной молекулы хромона. Данный процесс происхо- дит в течение единиц пикосекунд. После разрыва связи молекула переходит в нестационарное состояние $B$ : изменившееся распределение электронной плотности инициирует изменение обобщенной координаты молекулы, т.е. смещение ядер в молекуле к новым положениям, соответствующим минимуму потенциальной энергии, и развороту цепочки в молекуле в течение временного промежутка порядка единиц наносекунд.

\section{Заключение}

В настоящей работе были измерены спектры поглощения переходного состояния молекул хромонов LHC480 с временным разрешением до сотен фемтосекунд при возбуждении импульсом излучения с длиной волны $320 \mathrm{~nm}$. Динамика релаксации наведенных спектров поглощения описывается двухэкспоненциальной функцией с временными постоянными порядка 1.5 ps и $1.5 \mathrm{~ns}$.

В области 450-500 nm наблюдается характерное смещение пика поглощения в более длинноволновую область в диапазоне задержек между зондирующим и возбуждающим импульсами от 1 до 5 ps. Для наблюдаемой динамики спектров поглощения была предложена модель процесса трансформации молекулы и соответствующее схематичное изображение энергетической диаграммы. В предложенной модели временам порядка пикосекунд соответствует разрыв связи в одном из углеродных колец и переход молекулы в нестационарное возбужденное состояние $B$ с изменившемся распределением электронной плотности. В возбужденном состоянии атомы в молекуле смещаются к новым положениям с минимум потенциальной энергии, и в результате в течение времени порядка единиц наносекунд происходит трансформация молекулы в конечное люминесцирующее состояние.

Дополнительную информацию о динамике фотопреобразования, в том числе при двухфотонном возбуждении молекулы, позволят получить дополнительные эксперименты методами „рump-repump-probe“ спектроскопии, в которой используются два независимых возбуждающих импульса, с одновременной регистрацией сигнала люминесценции молекулы в процессе фотопреобразования.

\section{Список литературы}

[1] Kiyko V.V. Patent US8455079 B2 USA. 2013.

[2] Krayushkin M., Levchenko K., Yarovenko V. // ARKIVOC. 2009.

[3] Martynov I.Y., Barachevsky V.A., Ayt A.O., Kobeleva O.I., Valova T.M., Levchenko K.S., Yarovenko V.N., Krayushkin M.M. // Opt. Mater. (Amst). 2014. V. 37. P. 488-492. doi 10.1016/j.optmat.2014.07.011

[4] Ayt A., Barachevsky V.A., Kobeleva O.I., Valova T.M., Gagarskiy S.V, Kiyko V.V, Sergeev A.N., Veniaminov A.V, Zakharov V.V, Krayushkin M., Iglev H. // 2014 International Conference Laser Optics. 2014. P. 1. doi 10.1109/LO.2014.6886252 
[5] Ayt A.O., Barachevsky V.A., Duensing A., Fomicheva Y.Y., Gagarskiy S.V, Iglev H., Kiyko V.V, Krayushkin M.M., Sergeev A.N., Veniaminov A.V, Zakharov V.V // Opt. Quantum Electron. 2017. V. 49, N 2. P. 72. doi 10.1007/s11082-017-0900-3

[6] Ishibashi Y., Fujiwara M., Umesato T., Saito H., Kobatake S., Irie M., Miyasaka H. // J. Phys. Chem. C. 2011. V. 115. N 10. P. 4265-4272. doi 10.1021/jp112370a

[7] Ward C.L., Elles C.G. // J. Phys. Chem. A. 2014. V. 118. N 43. P. 10011-10019. doi 10.1021/jp5088948

[8] Irie M., Sayo K. // J. Phys. Chem. 1992. V. 96. N 19. P. $7671-$ 7674. doi 10.1021/j100198a035

[9] Hiroshi M., TaNahiro N., Masataka M., Akira I., Tamai N., Irie M. // J. Phys. Chem. A. 2002. V. 106. N 35. P. 8096-8102. doi 10.1021/JP0206626

[10] Miyasaka H., Ito S., Ishibashi Y. // Photon-Working Switches. 2017. P. 225-235. doi 10.1007/978-4-431-56544-4_11

[11] Fukaminato T., Doi T., Tanaka M., Irie M. // J. Phys. Chem. C. 2009. V. 113. N 27. P. 11623-11627. doi 10.1021/jp902880d

[12] Shim S., Eom I., Joo T., Kim E., Kim K.S. // J. Phys. Chem. A. 2007. doi 10.1021/JP0715528

[13] Tamai N., Miyasaka H. // Chem. Rev. 2000. V. 100. N 5. P. 1875-1890. doi 10.1021/cr9800816

[14] Murakami M., Miyasaka H., Okada T., Kobatake S., Irie M. // J. Am. Chem. Soc. 2004. V. 126. N 45. P. 14764-14772. doi $10.1021 / \mathrm{ja} 049177+$

[15] Ishibashi Y., Mukaida M., Falkenström M., Miyasaka H., Kobatake S., Irie M. // Phys. Chem. Chem. Phys. 2009. V. 11. N 15. P. 2640. doi 10.1039/b818591c 